\title{
Development of an Enzyme-Linked Immunosorbent Assay System to Determine the Presence of Antibodies Specific for Taxane Structures
}

\author{
Satoshi Muto, ${ }^{*}, a$ Yuichi Kouyama, ${ }^{a}$ Yuusaku Yokoyama, ${ }^{b}$ Hiroaki Okuno, ${ }^{b}$ Toshihiro Ishil, ${ }^{c}$ \\ Toru Masaka, ${ }^{c}$ Yasuo Matsuzawa, ${ }^{d}$ Tatsuo Kawashima, ${ }^{d}$ and Koji Shirai ${ }^{e}$ \\ ${ }^{a}$ Department of Disease Analysis, Faculty of Pharmaceutical Sciences, Toho University; ${ }^{b}$ Department of Pharmaceutical \\ Sciences, Faculty of Pharmaceutical Sciences, Toho University; 2-2-1 Miyama, Funabashi, Chiba 274-8510, Japan: \\ ${ }^{c}$ Department of Pharmacy, Toho University Sakura Medical Center; ${ }^{d}$ Department of Respiratory Medicine, Toho \\ University Sakura Medical Center; and ${ }^{e}$ The Center of Diabetes, Endocrinology, and Metabolism, Toho University Sakura \\ Medical Center; 564-1 Shimoshizu, Sakura, Chiba 285-8741, Japan.
}

Received January 6, 2011; accepted January 25, 2011; published online February 3, 2011

\begin{abstract}
Taxanes, which are widely used in treatment of numerous cancer types, are well-known to induce hypersensitivity reactions (HSR), especially in the case of paclitaxel. Although the cause of the HSR is commonly thought to be a non-immunological direct effect of the diluent which is used to dissolve paclitaxel, some reports suggest the possibility of the presence of an immunological reaction to the common taxane structure. The aim of this study was to establish a method to determine the presence of anti-taxane antibodies in body fluids of patients who have previously received paclitaxel, in order to estimate the risk of the occurrence of HSR to other taxane compounds, such as docetaxel. To prepare an enzyme-linked immunosorbent assay (ELISA) plate for determining taxanes, 10-deacetylbaccatin III (DAB) was first succinylated by use of dimethylaminopyridine and succinic anhydride in dried pyridine. After the succinylation reaction, three different products were obtained, and these were confirmed as 7-succinoyl DAB (7-DAB), 10-succinoyl DAB (10-DAB), and 7,10-disuccinoyl DAB (7,10-DAB) by ${ }^{1} \mathrm{H}-\mathrm{NMR}$ analysis. Each of these three products was conjugated with bovine serum albumin (BSA), and adsorbed on an ELISA plate. By using a commercially available anti-taxane monoclonal antibody as a model antibody, the detection limit of the anti-taxane antibodies on the 7-DAB-BSA-, 10-DAB-BSA-, and 7,10-DAB-BSAconjugated ELISA plate was estimated as $0.3,1$ and $10 \mathrm{ng} / \mathrm{ml}$, respectively. The ELISA system established in this study may therefore be useful for estimating the risk of HSR to taxanes in a patient prior to the use of these drugs.
\end{abstract}

Key words taxane; antibody; enzyme-linked immunosorbent assay; deacethylbaccatin; succinylation; hypersensitivity

Paclitaxel and docetaxel, which generally refered to as taxanes since they have a common taxane structure, are widely used in the treatment of numerous cancer types, including breast and ovarian cancers. ${ }^{1,2)}$ They are also known to induce hypersensitivity reactions (HSRs). Especially in the case of paclitaxel, pretreatment with dexamethasone and histamine receptor antagonists is recommended to avoid the occurrence of severe HSRs. ${ }^{3-5)}$

Docetaxel, which is more soluble than paclitaxel and has been used as an alternative taxane, is commonly considered an option for patients who have experienced severe HSRs to paclitaxel. ${ }^{6,7)}$ However, the incidence of allergic reactions to docetaxel is reported in the range of $10-20 \%$, especially in patients previously treated with paclitaxel, while the incidence is as low as $2 \%$ in the taxane-naive population. ${ }^{8)}$

The cause of the HSRs is commonly thought to be a nonimmunological direct effect of a diluent, Cremophor EL, which is used to dissolve the paclitaxel. ${ }^{9-11)}$ However, some reports suggest the possibility of the presence of an immunological reaction to the common taxane structure. ${ }^{8,12)}$ Indeed, Dizon et $a l .{ }^{8)}$ reported that when 10 patients experiencing severe HSRs to paclitaxel were subsequently treated with docetaxel, 9 of them also experienced severe HSRs, for an overall cross sensitivity rate of $90 \%$.

Since most of the patients with HSRs to taxanes present with symptoms of a Type I hypersensitivity reaction, such as dyspnea, hypotension, bronchospasm, and urticaria, immunoglobulin E (IgE) is speculated to be involved in the occurrence of HSRs to taxanes. Therefore, it is useful to examine the presence of anti-taxane $\operatorname{IgE}$ in the body fluids of the patients who have previously received paclitaxel, in order to estimate their risk of HSRs to docetaxel. However, such a method to determine $\operatorname{IgE}$ antibodies against the taxane structures in human serum has not yet been developed.

We herein report the development of a simple and sensitive enzyme-linked immunosorbent assay (ELISA) system for determining anti-taxane antibodies, and attempt to show that this method is clinically applicable for measuring the anti-taxane specific IgE in patients, thereby helping to prevent the development of HSRs.

\section{MATERIALS AND METHODS}

Materials 10-Deacetylbaccatin III (DAB), bovine serum albumin (BSA), and mouse monoclonal antibody against taxane structures (class: IgG1, clone: 8A10) were purchased from Wako Pure Chemicals (Osaka, Japan), Sigma (U.S.A.), and Hawaii Biotec Inc. (U.S.A.), respectively. Human IgE (derived from a myeloma patient), an anti-human IgE mouse monoclonal antibody, a biotinylated anti-human IgE antibody, and a peroxidase (POD)-conjugated streptavidin were purchased from Yamasa (Chiba, Japan), HyTest (Finland), KPL (U.S.A.), and Wako (Osaka, Japan), respectively. All other reagents were obtained from Wako.

Succinylation of 10-Deacethylbaccatin III In order to immobilize taxanes on the ELISA plates, we set up the strategies shown in Fig. 1. The succinylation of 10-deacethylbaccatin III (DAB) was first carried out according to the 

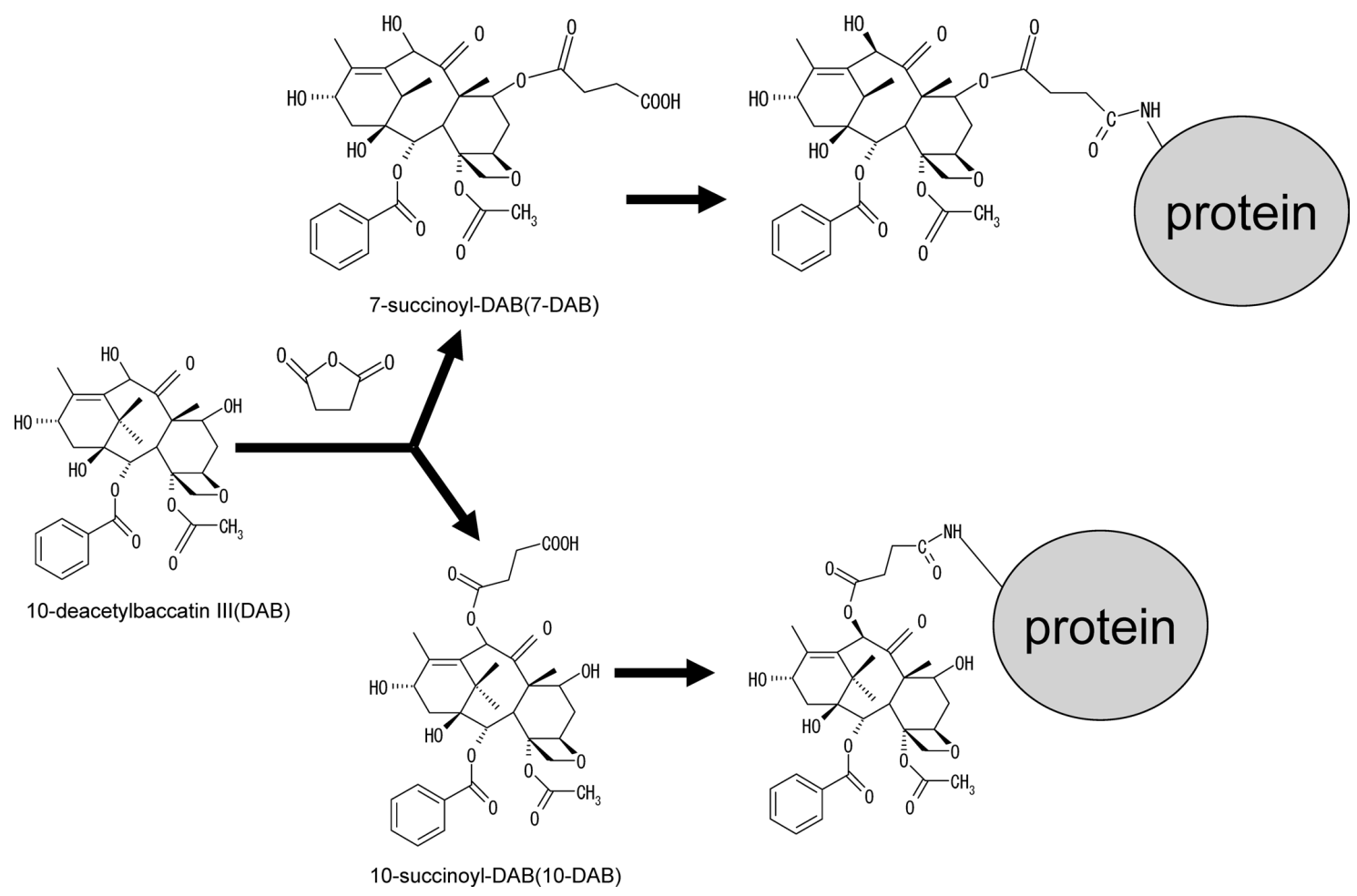

Fig. 1. Strategy for the Production of Taxane-Protein Conjugates

This figure shows the case where the succinylation has occurred at the $\mathrm{C}-7$ or $\mathrm{C}-10$ positions of DAB as an example.

method reported by Guo et al., ${ }^{13)}$ with a slight modification. A mixture of dimethylaminopyridine $(3.9 \mathrm{mg}, 31.9 \mu \mathrm{mol})$ and succinic anhydride $(55.8 \mathrm{mg}, 558 \mu \mathrm{mol})$ was dissolved in dry pyridine $(0.75 \mathrm{ml})$. An aliquot of this solution $(0.56 \mathrm{ml})$ was added to DAB $(55.4 \mathrm{mg}, 102 \mu \mathrm{mol})$, and the mixture were stirred at room temperature for $7.5 \mathrm{~h}$ under argon atmosphere. Next, the reaction mixture was evaporated to remove pyridine completely by repeated azeotropic distillation with benzene and cyclohexane. The resulting residue was dissolved in a small amount of methanol and subjected to preparative TLC on silica gel using $\mathrm{CHCl}_{3} / \mathrm{CH}_{3} \mathrm{OH}(9: 1)$ as the mobile phase. The succinylated products separated by TLC were extracted with methanol, and the structures were confirmed by ${ }^{1} \mathrm{H}-\mathrm{NMR}$.

Conjugation of Succinylated DABs with BSA Succinylated DABs were conjugated with BSA, according to the method described by Guo et al. ${ }^{13)}$ with a slight modification. Briefly, $9 \mathrm{mg}$ of each of the succinylated DABs (in the case of 7,10-disuccinoyl-DAB, $4.5 \mathrm{mg}$ was used) were dissolved in $1 \mathrm{ml}$ of dimethyl sulfoxide, and then tributylamine $(50 \mu \mathrm{l})$ was added, and the solution was cooled at $4{ }^{\circ} \mathrm{C}$. After $30 \mathrm{~min}$, isobutylchloroformate $(25 \mu \mathrm{l})$ was added, and the mixture was left at $4{ }^{\circ} \mathrm{C}$ for a further $30 \mathrm{~min}$. The mixture was added dropwise to a solution of BSA ( $25 \mathrm{mg}$ BSA was dissolved in $3 \mathrm{ml}$ of $10 \mathrm{~mm}$ sodium carbonate buffer, $\mathrm{pH} 9.5$, and cooled to $4{ }^{\circ} \mathrm{C}$ ). The $\mathrm{pH}$ was immediately neutralized by adding $1 \mathrm{M}$ sodium phosphate buffer, $\mathrm{pH} 7.2$, and then the mixture was left overnight at $4{ }^{\circ} \mathrm{C}$. To remove the remaining unreacted chemicals, the reaction mixture was dialyzed by a repeated centrifugation and concentration procedure using an Amicon Ultra filter unit (cut off molecular weight (MW) 10000) with $10 \mathrm{~mm}$ Tris-buffered saline ( $\mathrm{pH}$ 7.4) containing $0.1 \% \mathrm{NaN}_{3}$ (TBS/azide). The protein concentration of the solution thus obtained was determined by using a Bio-Rad Protein Assay (Bio-Rad), and the solution was used as DAB-conjugated BSA in the following experiments.

ELISA for Determining Anti-taxane Antibodies DABconjugated BSA-coated ELISA plates were prepared as follows. A $100 \mu \mathrm{l}$ aliquot of DAB-conjugated BSA solution $(10-100 \mu \mathrm{g} / \mathrm{ml})$ was added to each well of a 96-well ELISA plate (Iwaki) and left overnight at $4{ }^{\circ} \mathrm{C}$. After the solution in the wells was discarded, $350 \mu \mathrm{l}$ of $1 \% \mathrm{BSA} / \mathrm{TBS} /$ azide was added to the wells and left overnight again at $4{ }^{\circ} \mathrm{C}$. Immediately before the assay, the solution in the wells was discarded, and thereafter the wells were rinsed 3 times with TBS containing $0.05 \%$ Tween 20 (TBS/Tween) and once with TBS/ azide.

ELISA for the determination of anti-taxane antibodies was performed as follows. A mouse monoclonal antibody against taxanes was serially diluted with $1 \% \mathrm{BSA} / \mathrm{TBS} / \mathrm{Tw}$ een/azide, and $100 \mu \mathrm{l}$ of the solution was added to each well of the ELISA plate which was coated with DAB-conjugated BSA. After $1 \mathrm{~h}$, the solution was removed, and the wells were washed 3 times with TBS/Tween and once with TBS, and then $100 \mu \mathrm{l}$ of POD-conjugated anti-mouse $\operatorname{IgG}$ antibody ( $2 \mu \mathrm{g} / \mathrm{ml}$ in $1 \% \mathrm{BSA} / \mathrm{TBS} /$ Tween) was added to the wells. After $1 \mathrm{~h}$, the solution was removed and the wells were washed 3 times with TBS/Tween and once with TBS. Thereafter, $100 \mu \mathrm{l}$ of $3,3^{\prime}, 5,5^{\prime}$-tetramethylbenzidine solution (TMB solution, Wako) was added to the wells as a substrate for POD, and the plate was incubated until an appropriate color was developed. The reaction was stopped by adding $25 \mu \mathrm{l}$ of $1 \mathrm{M} \mathrm{H}_{2} \mathrm{SO}_{4}$, and the absorbance of each well was read with a microplate reader (Bio-Rad, Model 550), using a sample wavelength fixed at $450 \mathrm{~nm}$ and a reference wavelength at $655 \mathrm{~nm}$. 
Table 1. $\quad{ }^{1}$ H-NMR Analysis for the Succinylation Products of 10-Accetylbaccatin III (DAB)

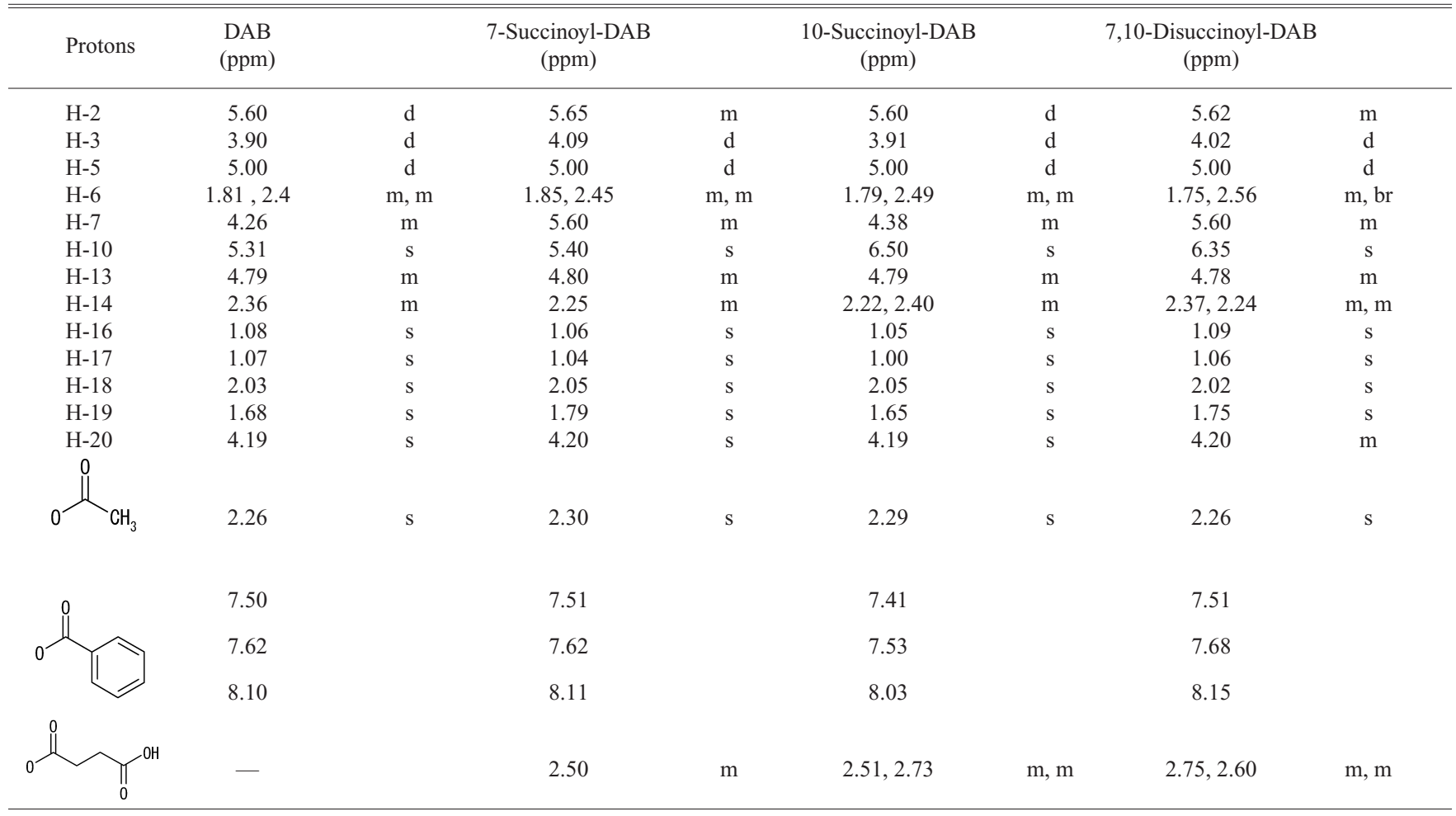

ELISA for Determining Human IgE To prepare the ELISA plate for the determination of human IgE, $100 \mu \mathrm{l}$ of anti-human IgE mouse monoclonal antibody $(10 \mu \mathrm{g} / \mathrm{ml}$ in TBS/azide) was added to each well of a 96-well ELISA plate and left overnight at $4{ }^{\circ} \mathrm{C}$. After the solution in the wells was discarded, $350 \mu \mathrm{l}$ of $1 \% \mathrm{BSA} / \mathrm{TBS} /$ azide was added to the wells and left overnight again at $4{ }^{\circ} \mathrm{C}$. Immediately before the assay, the solution in the wells was discarded, and thereafter, the wells were rinsed 3 times with TBS/Tween and once with TBS/azide.

The ELISA for the determination of human IgE was performed as follows: A human IgE was serially diluted with $1 \% \mathrm{BSA} / \mathrm{TBS} / \mathrm{Tw}$ ween/azide, and $100 \mu \mathrm{l}$ of the solution was added to each well of the ELISA plate, which was coated with the anti-human IgE antibody. After $1 \mathrm{~h}$, the solution was removed, and the wells were washed 3 times with TBS/ Tween and once with TBS, and then $100 \mu \mathrm{l}$ of biotinylated anti-human IgE antibody $(0.5 \mu \mathrm{g} / \mathrm{ml}$ in $1 \% \mathrm{BSA} / \mathrm{TBS} / \mathrm{Tw}$ een/ azide) was added to the wells. After $1 \mathrm{~h}$, the solution was removed and the wells were washed 3 times with TBS/Tween and once with TBS, and then $100 \mu \mathrm{l}$ of POD-conjugated streptavidin $(0.5 \mu \mathrm{g} / \mathrm{ml}$ in $1 \% \mathrm{BSA} / \mathrm{TBS} /$ Tween $)$ was added to the wells. After $1 \mathrm{~h}$, the solution was removed and the wells were washed 3 times with TBS/Tween and once with TBS. Thereafter, $100 \mu \mathrm{l}$ of TMB solution was added to the wells, and the plate was incubated until an appropriate color was developed. The reaction was stopped by adding $25 \mu \mathrm{l}$ of $1 \mathrm{M}$ $\mathrm{H}_{2} \mathrm{SO}_{4}$, and the absorbance of each well was read with a microplate reader, using a sample wavelength fixed at $450 \mathrm{~nm}$ and a reference wavelength at $655 \mathrm{~nm}$.

\section{RESULTS}

Synthesis of Succinylated DAB After the succinylation of DAB, three different products were obtained by preparative TLC. Two of them were confirmed as 7-succinoyl-DAB and 10-succinoyl-DAB, as previously reported by Guo et al., by ${ }^{1} \mathrm{H}-\mathrm{NMR}$ analysis. The structure of the other product that was newly isolated in the present study, was confirmed to be 7,10-succinoyl-DAB as follows; 1) on mass spectroscopy, the molecular ion $(811 \mathrm{~m} / \mathrm{z})$ showed the addition of two succinoyl moiety to DAB (data not shown), 2) on the ${ }^{1} \mathrm{H}-\mathrm{NMR}$ spectrum, the peaks at 5.60 and $6.35 \mathrm{ppm}$ of the third product could be assigned to the protons attached at $\mathrm{C} 7-$ and $\mathrm{C} 10$ based on the comparison of the NMR spectra of 7-succinoylDAB and 10-succinoyl-DAB (Table 1).

The yields of 7-succinoyl-DAB, 10-succinoyl-DAB, and 7,10-disuccinoyl-DAB were 23,42 , and $19 \%$, respectively.

Detection Limits of the Anti-taxane Antibodies on the Three Different DAB-BSA Conjugates Coated on the ELISA Plate To examine the capability of these three succinoyl-DAB products to be recognized by anti-taxane antibodies, these were conjugated with BSA and adsorbed onto ELISA plates. Commercially available anti-taxane monoclonal antibodies, which we used in this study, were observed to significantly bind to all of these DAB-BSA coated wells, but not to BSA coated control wells (data not shown). We next examined the detection limits of the anti-taxane antibodies on these three DAB-coated ELISA plates.

As shown in Fig. 2, anti-taxane antibodies were bound to all three types of DAB-coated wells, dependent on the amounts of antibody added. When an absorbance of 0.02 was considered to be an identification limit, the detection limit of the anti-taxane antibodies on 7-succinoyl-DAB-, 10-succinoyl-DAB-, and 7,10-disuccinoyl DAB-coated wells were $0.3,1$ and $10 \mathrm{ng} / \mathrm{ml}$, respectively.

Determination of Human IgE To evaluate whether the ELISA system, as a model for the determination of anti-tax- 
(A)

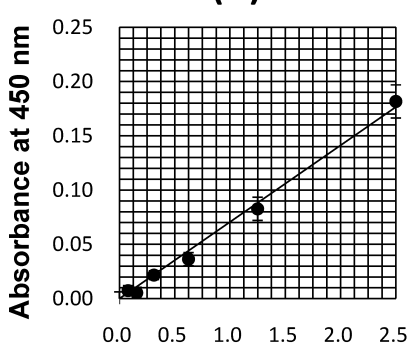

(B)

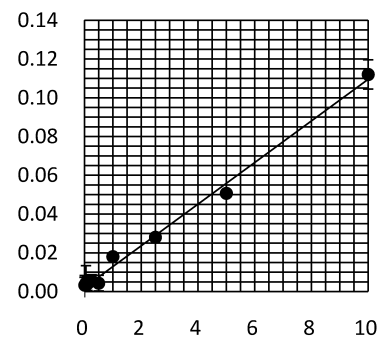

(C)

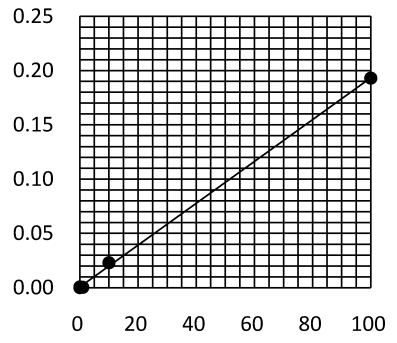

Anti-taxane antibodies $(\mathrm{ng} / \mathrm{ml})$

Fig. 2. Detection Limits of Anti-taxane Monoclonal Antibody (8A10) on Three Different DAB-Coated Wells

Three succinoyl-DAB products were conjugated with BSA and adsorbed on ELISA plates. Anti-taxane mouse monoclonal antibodies were serially diluted with $1 \% \mathrm{BSA} / \mathrm{TBS} /$ Tween/azide, and $100 \mu \mathrm{l}$ of the solution was added to each well of the DAB-coated ELISA plates. Then the bound antibodies were detected by use of POD-conjugated anti-mouse IgG antibodies and substrate for POD (see Materials and Methods). The data were expressed as the mean absorbance at 450 nm of triplicate wells. (A) 7-DABcoated plate, (B)10-DAB-coated plate, (C) 7,10-DAB-coated plate.

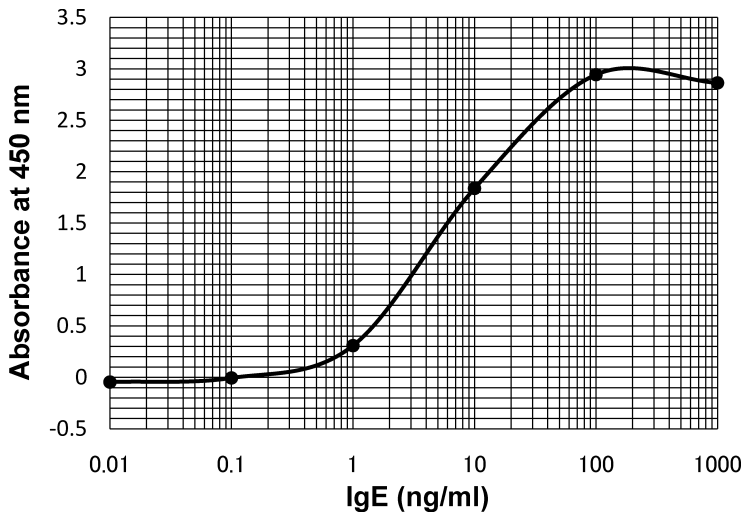

Fig. 3. Standard Curve for the Determination of Human IgE on Antihuman IgE Coated Plates

Human IgE samples were serially diluted with $1 \% \mathrm{BSA} / \mathrm{TBS} /$ Tween/azide, and $100 \mu \mathrm{l}$ of the solution was added to each well of anti-human IgE mouse monoclonal antibodycoated ELISA plates. Then, the bound IgE was detected by use of the biotinylated antihuman IgE antibody and POD-conjugated streptavidin (see Materials and Methods) The data were expressed as the mean absorbance at $450 \mathrm{~nm}$ of triplicate wells.

ane $\mathrm{IgG}$, is applicable to determine the human $\mathrm{IgE}$ antibodies against taxanes, we examined the detection limit of human IgE using an anti-human IgE-coated ELISA plate. As shown in Fig. 3, human IgE was detected at levels as low as $1 \mathrm{ng} / \mathrm{ml}$ by ELISA using biotinylated anti-human $\operatorname{IgE}$ antibodies and POD-conjugated streptavidin.

\section{DISCUSSION}

In this study, we established a simple and sensitive ELISA system for the determination of anti-taxane antibodies. Although our strategy to prepare the taxane-coated ELISA plate was almost the same as the method described by Guo et al., ${ }^{13)}$ we aimed to use the taxane-coated ELISA plate to detect anti-taxane antibodies in human serum, whereas Guo et al. used such ELISA plates to detect taxane compounds in Taxus sp. plants and in tissue cultures. Other researchers ${ }^{14-18)}$ have also reported the use of anti-taxane antibodies to detect or purify the taxane compounds from plants, but there have so far been few reports that have used such a taxane-coated ELISA plate to detect anti-taxane antibodies. The exception is the report by Vanhaelen et al., ${ }^{19)}$ who showed the presence of anti-taxane antibodies in healthy individuals. Thus, the present study is the first to try to apply the taxane-coated
ELISA plate for clinical use to prevent HSRs.

At the begining of our experiments on the succinylation of DAB, we expected to obtain 4 succinylated products of DAB, because DAB has 4 hydroxyl moieties at the $\mathrm{C}-1, \mathrm{C}-7, \mathrm{C}-10$, and $\mathrm{C}-13$ positions. Unfortunately, we could not obtain 1succinoyl DAB and 13-succinoyl DAB, similar to the report of Guo et al. ${ }^{13)}$ The reason for our failure to obtain these compounds may be the low reactivity of these positions. However, we identified a newly-isolated 7,10-disuccinoyl$\mathrm{DAB}$ from the reaction mixture, in addition to the 7-succinoyl-DAB and 10-succinoyl-DAB that had been isolated previously. ${ }^{13)}$ The reason for the formation of 7,10-disuccinoylDAB might have been the longer reaction time and larger molar ratio of succinic anhydride compared to Guo's report.

We found that the detection limits of anti-taxane-antibodies differed among the three succucinoyl DABs-coated plates, perhaps as a result of the antigen-specificity of the anti-taxane monoclonal antibody used in this study as a model antibody, or differences in the number of haptens that are presented on BSA. We suspected the latter because, in the coupling reaction of succinylated products (haptens) and $\mathrm{BSA}$, we reacted 7 -DAB or $10-\mathrm{DAB}$ with $\mathrm{BSA}$ at molar ratio of $40: 1$, but $7,10-\mathrm{DAB}$ was reacted with BSA at $20: 1$. Although we did not examine how many haptens are actually coupled to BSA after the coupling reaction, it is likely that more DAB molecules are presented on 7-DAB-BSA and 10DAB-BSA conjugates than 7,10-DAB-BSA conjugates. Furthermore, since disuccinoyl DAB was able to bind to BSA with two succinoyl moieties and cause steric hindrance, it might be possible that anti-taxane antibodies cannot bind as easily to 7,10-DAB-coated plates, thus resulting in a worse detection limit. It might be necessary to consider the coupling method to improve the detection sensitivity of this assay system in the future.

The cause of the hypersensitivity reactions (HSRs) induced by paclitaxel is commonly thought to be a nonimmunological direct effect of the diluent, Cremophor EL, which is used to dissolve paclitaxel. Therefore, especially in the case of paclitaxel, pre-treatment with dexamethasone and histamine receptor antagonists is recommended to avoid the occurrence of severe HSRs. Based on the hypothesized mechanism of sensitivity, a Cremophor-free, protein-stabilized, nanoparticle formulation of paclitaxel (Abraxane) was recently developed to help decrease HSRs. ${ }^{20,21)}$ 
However, HSRs also appear after treatment with docetaxel, which does not contain Cremophor EL. Dizon et al. ${ }^{8)}$ reported that when 10 patients experiencing severe HSRs to paclitaxel were subsequently treated with docetaxel, 9 of them also experienced severe HSRs for an overall cross sensitivity rate of $90 \%$. This suggests that there may be an immunological reaction to the common taxane structure.

Prieto and Pineda ${ }^{22)}$ recently demonstrated the presence of anti-paclitaxel antibodies in the serum of a patient who developed HSRs after receiving paclitaxel by use of IgE dotblot assay. This indicates that anti-taxane antibodies could be involved in the HSRs to taxane compounds.

Therefore, in order to apply our DAB-coated ELISA plate to clinical use, we next developed a sensitive ELISA system for the determination of human IgE. In this system, human IgE could be detected at levels as low as $1 \mathrm{ng} / \mathrm{ml}$. This indicates that if a clinical sample contains anti-taxane $\operatorname{IgE}$ of $1 \mathrm{ng} / \mathrm{ml}$ or more, we will be able to detect it by using the combination of the DAB-coated ELISA plate and human IgE detection system (biotinylated anti-human IgE antibodies and POD-conjugated streptavidin).

Unfortunately, we were unable to demonstrate the presence of anti-taxane antibodies in patient serum yet, because we did not have access to an appropriately affected patient. However, we believe that the ELISA system established in this study will be clinically applicable for determining the presence of anti-taxane specific IgE, and would be useful for estimating the risk of HSRs in patients who have previously used taxane compounds.

Acknowledgements We thank Dr. Yohko Sakamoto and Dr. Katsuyoshi Mitsunaga for their valuable technical assistance in performing the NMR analysis and mass spectrometry.

\section{REFERENCES}

1) Wiernik P. H., Schwartz E. L., Strauman J. J., Dutcher J. P., Lipton R. B., Paietta E., Cancer Res., 47, 2486-2493 (1987).
2) Foa R., Norton L., Seidman A. D., Int. J. Clin. Lab. Res., 24, 6-14 (1994).

3) Peereboom D. M., Donehower R. C., Eisenhauer E. A., McGuire W. P., Onetto N., Hubbard J. L., Piccart M., Gianni L., Rowinsky E. K., J. Clin. Oncol., 11, 885-890 (1993).

4) Boehm D. K., Maksymiuk A. W., J. Natl. Cancer Inst., 88, 463-465 (1996).

5) Markman M., Kennedy A., Webster K., Peterson G., Kulp B., Belinson J., J. Cancer Res. Clin. Oncol., 125, 427-429 (1999).

6) Lokich J., Anderson N., Ann. Oncol., 9, 573 (1998).

7) Bernstein B. J., Ann. Pharmacother, 34, 1332-1335 (2000).

8) Dizon D. S., Schwartz J., Rojan A., Miller J., Pires L., Disilvestro P., Gordinier M. E., Moore R., Granai C. O., Legare R. D., Gynecol. Oncol., 100, 149-151 (2006).

9) Decorti G., Bartoli K. F., Candussio L., Baldini L., Anticancer Res., 16, 317-320 (1996).

10) Szebeni J., Muggia F. M., Alving C. R., J. Natl. Cancer Inst., 90, 300-306 (1998).

11) Szebeni J., Alving C. R., Savay S., Barenholz Y., Priev A., Danino D., Talmon Y., Int. Immunopharmacol., 1, 721-735 (2001).

12) Denman J. P., Gilbar P. J., Abdi E. A., J. Clin. Oncol., 20, 2760-2761 (2002).

13) Guo Y., Jaziri M., Diallo B., Vanhaelen-Fastre R., Zhiri A., Vanhaelen M., Homès J., Bombardelli E., Biol. Chem. Hoppe Seyler, 375, 281287 (1994).

14) Jaziri M., Diallo B. M., Vanhaelen M. H., Vanhaelen-Fastre R. J., Zhiri A., Bécu A. G., Homes J., J. Pharm. Belg., 46, 93 -99 (1991).

15) Grothaus P. G., Bignami G. S., O’Malley S., Harada K. E., Byrnes J. B., Waller D. F., Raybould T. J., McGuire M. T., Alvarado B., J. Nat. Prod., 58, 1003-1014 (1995).

16) Guo Y., Vanhaelen-Fastré R., Diallo B., Vanhaelen M., Jaziri M., Homes J., Ottinger R., J. Nat. Prod., 58, 1015-1023 (1995) .

17) Durzan D. J., Ventimiglia F., Havel L., Acta Astronaut., 42, 455-463 (1998).

18) Theodoridis G., Haasnoot W., Cazemier G., Schilt R., Jaziri M., Diallo B., Papadoyannis I. N., de Jong G. J., J. Chromatogr. A, 948, 177-185 (2002).

19) Vanhaelen M., Duchateau J., Vanhaelen-Fastre R., Jaziri M., Planta Med., 68, 36- 40 (2002).

20) Ibrahim N. K., Desai N., Legha S., Soon-Shiong P., Theriault R. L., Rivera E., Esmaeli B., Ring S. E., Bedikian A., Hortobagyi G. N., Ellerhorst J. A., Clin. Cancer Res., 8, 1038-1044 (2002).

21) Fader A. N., Rose P. G., Int. J. Gynecol. Cancer, 19, 1281-1283 (2009).

22) Prieto Garcia A., Pineda de la Losa F., J. Investig. Allergol. Clin. Immunol., 20, 170-171 (2010). 